\title{
Educación ambiental en instituciones educativas de Latinoamérica: revisión sistémica
}

\section{Environmental education in educational institutions in Latin America: a systemic review \\ Sandra Pizango ${ }^{1}$}

\section{RESUMEN}

La Educación Ambiental en las instituciones educativas cumple una función fundamental, a nivel del sistema formativo de toda la humanidad, ya que incide en la enseñanza y aprendizaje con una sociedad ambientalmente solidaria y sostenible, capaz de mitigar los problemas medioambientales. Se trabajó con el método PRISMA, Bajo este escenario, los objetivos de dicho artículo fueron la descripción del estado de las investigaciones sobre EA así como, analizar las premisas conceptuales y los resultados obtenidos referente a EA en las Instituciones Educativas de Latinoamérica, para ello se realizó una revisión sistémica en español y portugués utilizando las siguientes bases de datos como Redalyc, Cielo, scopus, Dialnet y Ebsco, se tomó en cuenta juicios de exclusión e inclusión, se trabajó con artículos publicados entre 2018 al 2021. Entre las conclusiones se obtuvo que a nivel Latinoamérica la EA en las Instituciones Educativas ha sido objeto de investigación por su importancia no solo en lo académico sino también en la vida personal del estudiante.

Palabras clave: Comportamiento sustentable, desarrollo sostenible, Medioambiental, prácticas ambientales

\section{ABSTRACT}

Environmental Education in educational institutions fulfills a fundamental function, at the level of the training system of all humanity, since it affects teaching and learning with an environmentally supportive and sustainable society, capable of mitigating environmental problems. Under this scenario, the objectives of said review article is to describe the state of research on Environmental Education, as well as to analyze the conceptual premises and the results obtained regarding EE in Educational Institutions in Latin America, for which a review was carried out. systemic in Spanish and Portuguese using the following databases such as Redalyc, Cielo, scopus, Dialnet and Ebsco. For this analysis, exclusion and inclusion trials were taken into account, working with articles published between 2018 and 2021. Among the conclusions it was obtained that in Latin America EE in Educational Institutions has been the subject of research due to its importance not only in terms of academic but also in the personal life of the student.

Keywords: Sustainable behavior, sustainable development, Environmental. 


\section{INTRODUCCIÓN}

Actualmente, venimos atravesando por diversos problemas ambientales a nivel global, como son el cambio climático, deforestación, pérdida de nuestra biodiversidad, suscitados en los diferentes ambientes y espacios, incluyendo a los gobiernos, empresas, medios de comunicación, Instituciones Educativas y partidos políticos (Arriola, 2017). Ante estos acontecimientos, la Educación Ambiental (EA) es una alternativa para afrontar $y$ frenar de forma adecuada la crisis ambiental antes mencionada, ya que interviene en las actitudes colectivas e individuales del ser humano, generando un soporte entre el hombre, ambiente y territorio (Herrera y Ríos, 2017; Quintana, 2017).

El termino EA, fue usado en conferencia Internacional sobre Medio Ambiente en Estocolmo en los años 70 (Silva et al, 2019; Rivera, 2017). Su prioridad es instruir e incentivar a la sociedad adaptar actitudes y comportamientos positivos, responsables y justas hacia el medio que les rodea, teniendo en cuenta las futuras generaciones con mejor calidad de vida (Ministerio de Educación, 2016; Salvatierra, 2018; Márquez et al., 2021). Además, cumple un rol fundamental en el desarrollo sostenible, con la formación de una sociedad ambientalmente equitativa (Estrada, Huaypar y Mamani, 2020). En definición, para Al-Naqbi y Alshannag (2018) la EA es la cercanía e involucramiento de la sociedad como estudiantes, docentes, padres y madres de familia en el avance educativo a fin de concientizar sobre el cuidado y preservación medioambiental.

Entonces, las Instituciones Educativas y los estudiantes son colaboradores principales, para construir cambios, ya que el aula es la segunda casa de formación, donde se puede promover la sensibilización y enseñanza con temas ambientales, en tanto, los docentes deben contribuir con instrumentos y acciones propicias, para encaminar al verdadero cambio de actitudes que se requiere hoy en día, bajo responsabilidad de preparar estudiantes competitivos y comprometidos con una educación de calidad (Pérez et al.,2019), en esa misma línea, para lograr la ansiada sostenibilidad medioambiental, deben ser abordados por los diferentes actores y demás instituciones, para ello, es fundamental los mecanismos utilizados en el proceso de enseñanza y concientización, para que un futuro la prácticas ambientales sea un hábito (Armesto y Montes de o, 2020).

En tanto, para afrontar dicha problemática, se crearon lineamientos legales para el desarrollo de programas y estrategias que impacte en su mitigación, es decir, se busca fomentar una EA en todo Latinoamérica (Espejel y Flores, 2017; Berdugo y Montaño, 2017). A nivel Latinoamérica, existen planes y políticas educativas para la adecuada formación en EA, que inicia desde la educación inicial hasta el nivel universitario (Mamani et al., 2020). Por ejemplo, en el Perú, tenemos el respaldo del Plan Nacional sobre Educación Ambiental (PLANEA) y Política Nacional sobre Educación Ambiental (PNEA), cuyo objetivo radica en generar impacto a través de adecuados comportamientos ambientales, donde la misma sociedad sea protagonista con las diversas prácticas medioambientales (Ministerio de Educación, 2016).

En virtud de lo expuesto, la presente revisión sistémica se justifica, en que hoy en día en las instituciones educativas de Latinoamérica, el modo de cuidar e interactuar con el ambiente, evidencia que la estrategia utilizada en la EA, no estaría siendo adecuada, pues probablemente solo se esté enseñando de manera teórica y los resultados no son eficientes, incidir que el logro de aprendizaje teórico es importante, pero no determinante, debido a que buscamos que aquellos conocimientos sean aplicados en los problemas ambientales con prácticas cotidianas (Estrada et al., 2020). Por ello, es de prioridad, la descripción de investigaciones referente a Educación Ambiental en Instituciones Educativas, permitiendo alcanzar sostenibilidad de nuestra biodiversidad, al generar compromiso 
en los estudiantes y familia en actividades ambientales (Yangali et al., 2021).

Finalmente, el objetivo del actual artículo de revisión sistémica, es describir el estado de investigaciones sobre EA en las instituciones Educativas, así mismo analizar las premisas conceptuales y los resultados obtenidos referente a EA en las instituciones educativas de Latinoamérica. Siendo, un rol fundamental el crear las bases de la cultura ambiental en los estudiantes y futuros profesionales fortaleciendo la conciencia ambiental (Cóndor, 2018).

\section{MATERIAL Y METODOS}

La presenteinvestigación, fue de revisión sistémica (Hernández et al, 2014). Tuvo como finalidad la descripción del estado de investigaciones sobre Educación ambiental, las cuales dieron lugar a esquematizar la información encontrada sobre artículos científicos indexados de gran impacto, permitiendo analizar los resultados de manera reflexiva (Pulido y Olivera, 2018).En tanto, para sistematizar la información referente a "Educación Ambiental" se seleccionaron, artículos publicados desde el 2018 al 2021 en español y portugués, se respaldó de acuerdo las siguientes bases de datos: Scopus, Cielo, Dialnet, Ebsco y Redalyc, con un total de 836 artículos encontrados.

Sin embargo, se excluyeron aquellos que no alcanzaron los criterios requeridos de inclusión como: estudios en Instituciones Educativas, estudios en Latinoamérica, investigaciones en español y portugués, artículos de acceso abierto e investigaciones de los años requeridos. Así mismo seexcluyeron: estudios aplicados en universidades, artículos teóricos y las investigaciones fuera de Latinoamérica. De acuerdo al método Prisma, donde se representa a través de una gráfica de las investigaciones estudiadas y seleccionadas (Peramo,2020), se obtuvieron como resultado un total de 12 artículos, las cuales cumplen con los objetivos planteados y conforman la plataforma para la revisión.

\section{Figura 1Desarrollo de Revisión Prisma}

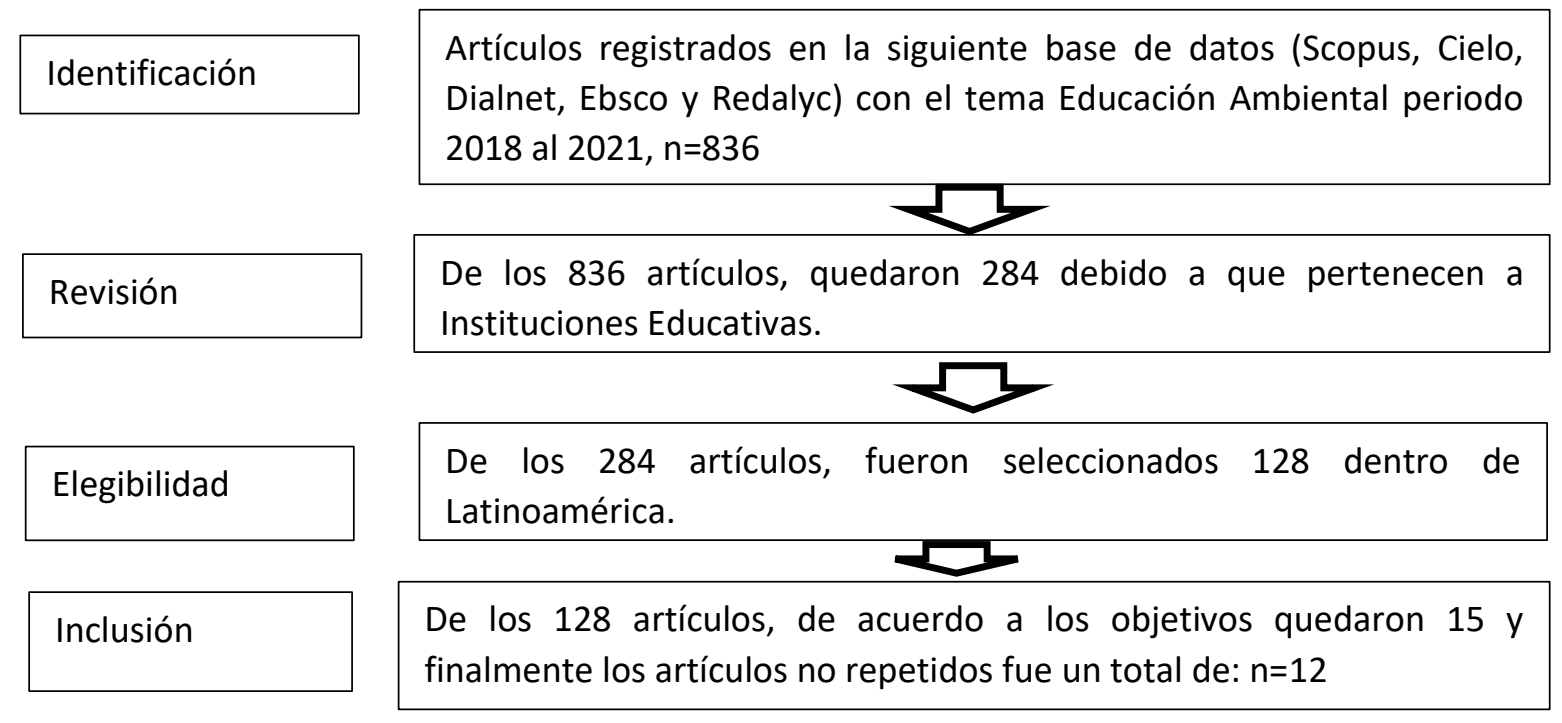

Nota: Detalle sobre el desarrollo de exclusión de las referencias en la base de datos de acuerdo al método PRISMA 
Tabla Nº 1. Resultado de Selección de los Repositorios

\begin{tabular}{|c|c|c|c|c|c|c|c|c|}
\hline Repositorio & Código & $\begin{array}{l}\text { Combinación } \\
\text { de búsqueda }\end{array}$ & Resultados & Temática & Región & Accceso & Objetivos & $\begin{array}{l}\text { Artículos no } \\
\text { repetidos }\end{array}$ \\
\hline \multirow[t]{2}{*}{ SCOPUS } & A1 & $\begin{array}{l}\text { EDUCACION } \\
\text { AMBIENTAL }\end{array}$ & 54 & 11 & 10 & 4 & 1 & \multirow[t]{2}{*}{2} \\
\hline & A2 & $\begin{array}{l}\text { Educação } \\
\text { Ambiental }\end{array}$ & 4 & 3 & 3 & 2 & 1 & \\
\hline \multirow{2}{*}{ CIELO } & B1 & $\begin{array}{l}\text { EDUCACION } \\
\text { AMBIENTAL }\end{array}$ & 24 & 13 & 7 & 4 & 3 & \multirow{2}{*}{4} \\
\hline & B2 & $\begin{array}{l}\text { Educação } \\
\text { Ambiental }\end{array}$ & 76 & 38 & 16 & 0 & 1 & \\
\hline \multirow{2}{*}{ DIALNET } & C1 & $\begin{array}{l}\text { EDUCACION } \\
\text { AMBIENTAL }\end{array}$ & 201 & 31 & 3 & 2 & 1 & \multirow{2}{*}{1} \\
\hline & C2 & $\begin{array}{l}\text { Educação } \\
\text { Ambiental }\end{array}$ & 154 & 43 & 13 & 4 & 0 & \\
\hline \multirow{2}{*}{ EBSCO } & D1 & $\begin{array}{l}\text { EDUCACION } \\
\text { AMBIENTAL }\end{array}$ & 132 & 47 & 31 & 7 & 4 & \multirow{2}{*}{5} \\
\hline & $\mathrm{D} 2$ & $\begin{array}{l}\text { Educação } \\
\text { Ambiental }\end{array}$ & 57 & 26 & 12 & 10 & 1 & \\
\hline \multirow{2}{*}{ REDALYC } & E1 & $\begin{array}{l}\text { EDUCACION } \\
\text { AMBIENTAL }\end{array}$ & 54 & 30 & 22 & 10 & 0 & \multirow{2}{*}{0} \\
\hline & E2 & $\begin{array}{l}\text { Educação } \\
\text { Ambiental }\end{array}$ & 80 & 42 & 11 & 11 & 0 & \\
\hline Total & & OTAL & 836 & 284 & 128 & 54 & 12 & 12 \\
\hline
\end{tabular}

En la tabla 1 se denota el proceso de selección; de acuerdo a los repositorios y el motor de búsqueda que fue (Educación Ambiental), se obtuvieron 836 artículos. Sin embargo, se seleccionaron las investigaciones en las Instituciones Educativas de Latinoamérica y los vinculados con los objetivos no repetidos fueron 12 .

\section{RESULTADOS}

1.1 Características de los artículos para la descripción del estado de las investigaciones sobre Educación Ambiental

Como resultado del desarrollo de revisión Prisma, se quedó con un total de doce (12) artículos encontrados en la base de datos Scopus, Ebsco, cielo y Dialnet. De estos, podemos observar que la mayoría es de procedencia colombiana. Así, se evidencia que Colombia ocupa el $42 \%$ con cinco (5) publicaciones, después tenemos a Brasil y Perú ambos con 25\% con tres (3) publicaciones cada uno, haciendo un total de seis (6) artículos rescatados para el presente análisis de revisión. Finalmente tenemos con $8 \%$ a Paraguay con un (1) artículo requerido para la descripción del estado de investigaciones.
Figura 2. Porcentaje de fuentes de acuerdo con la Región de procedencia

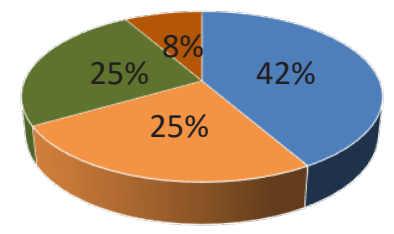

$$
\text { - Colombia - Brasil - Perú - Paraguay }
$$

En la Tabla 2 se evidencia los autores organizados según el año de publicación de las 12 investigaciones, en donde observamos, que gran parte de los estudios se centra en el año 2020, mostrando igualdad en el 2018 y 2019, siendo menor en el año 2021. 
Tabla $N^{\circ}$ 2. Autores clasificados por año de edición

\begin{tabular}{|r|r|l|}
\hline \multicolumn{1}{|c|}{ AÑO } & \multicolumn{1}{|c|}{ N $^{\circ}$} & \multicolumn{1}{c|}{ AUTORES } \\
\hline 2018 & 3 & $\begin{array}{l}\text { (Demoly y Dos santos,2018 ; Aparecida y Carlos ,2018 ; Gonzales } \\
\text { y Manrique,2018) }\end{array}$ \\
\hline 2019 & 3 & ( Calderón et al., 2019; Henao \& Sánchez, 2019; Alva,2019) \\
\hline 2020 & 5 & $\begin{array}{l}\text { (Miyazaki et al., 2020 ; Barrero,2020 ; Prosser et al., 2020 ; } \\
\text { Oliveira et al., 2020 ; Rivas et al., 2020) }\end{array}$ \\
\hline 2021 & 1 & (Espinoza y Ortiz,2021) \\
\hline
\end{tabular}

De acuerdo a la tabla 3 y figura 3, se ha logrado rescatar los variados enfoques, con los cuales se procesaron las investigaciones, así como el instrumento de recojo de datos aplicados para cada uno. De los artículos incluidos, se puede precisar que de los doce (12) artículos la mayoría fueron desarrolladas en base al método cualitativo con un total de nueve (9) investigaciones, dos (2) artículos siguieron el método cuantitativo, de igual manera un (1) artículo trabajo en base al método mixto.

Figura 3. Enfoque metodológico

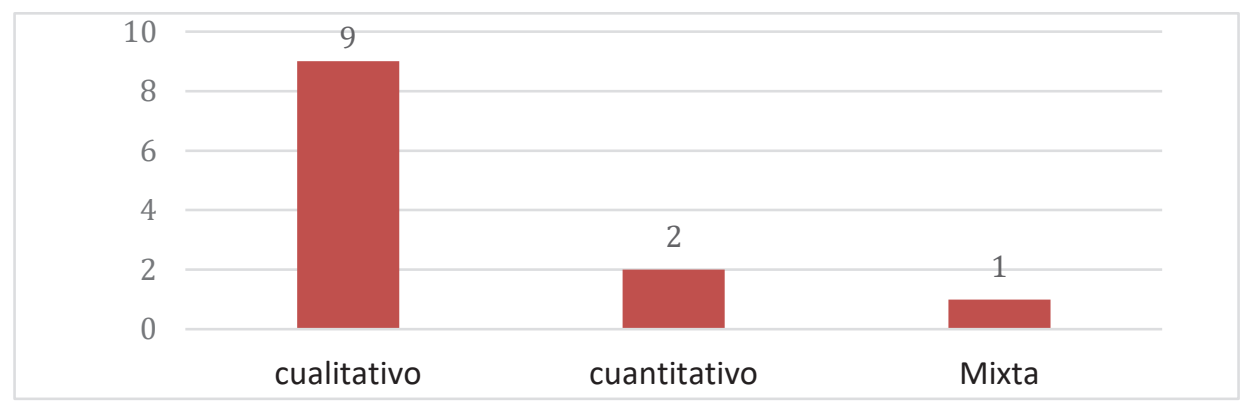

Tabla N ${ }^{\circ}$. Estrategias y enfoques Metodológicos

\begin{tabular}{|c|c|c|c|c|}
\hline REPOSITORIO & AUTOR Y AÑO & PAIS & METODOLOGIA & $\begin{array}{l}\text { INSTRUMENTO DE } \\
\text { RECOLECCIÓN DE } \\
\text { DATOS }\end{array}$ \\
\hline SCOPUS & $\begin{array}{l}\text { ( Calderon et } \\
\text { al., 2019) }\end{array}$ & colombia & cualitativa & Encuesta (Docentes) \\
\hline SCOPUS & $\begin{array}{l}\text { (Demoly y Dos } \\
\text { santos,2018) }\end{array}$ & Brazil & cualitativa & $\begin{array}{l}\text { Talleres fotograficos } \\
\text { (Estudiantes y docentes) }\end{array}$ \\
\hline CIELO & $\begin{array}{l}\text { (Espinoza y } \\
\text { ortiz,2021) }\end{array}$ & Peru & cualitativa & cuestionario (Docentes) \\
\hline CIELO & $\begin{array}{c}\text { (Miyazaki et al., } \\
\text { 2020) }\end{array}$ & Paraguay & cualitativa & $\begin{array}{c}\text { Entrevista y cuestionario } \\
\text { (personal } \\
\text { semiestructuradas) }\end{array}$ \\
\hline CIELO & $\begin{array}{c}\text { (Henao \& } \\
\text { Sánchez,2019) }\end{array}$ & colombia & cualitativa & $\begin{array}{c}\text { Analisis (de } 32 \\
\text { Proyectos Ambientales } \\
\text { Escolares) }\end{array}$ \\
\hline CIELO & $\begin{array}{l}\text { (Aparecida y } \\
\text { carlos ,2018) }\end{array}$ & Brasil & cualitativa & $\begin{array}{l}\text { Cuestionarios y } \\
\text { entrevistas ( Docentes y } \\
\text { coordinadores ) }\end{array}$ \\
\hline DIALNET & (Alva,2019) & Perú & cuantitativa & $\begin{array}{l}\text { Cuestionario } \\
\text { (Estudiantes) }\end{array}$ \\
\hline EBSCO & (Barrero,2020) & Colombia & cualitativa & Encuesta(Estudiantes) \\
\hline EBSCO & $\begin{array}{l}\text { (Prosser et al., } \\
\text { 2020) }\end{array}$ & colombia & cualitativa & Encuesta(Estudiantes) \\
\hline EBSCO & $\begin{array}{l}\text { (Oliveira et al., } \\
2020 \text { ) }\end{array}$ & Brasil & Mxta & Encuesta (Docentes) \\
\hline EBSCO & $\begin{array}{l}\text { (Rivas et al., } \\
\text { 2020) }\end{array}$ & colombia & cualitativa & Encuesta(Estudiantes) \\
\hline EBSCO & $\begin{array}{c}\text { (Gonzales y } \\
\text { Manrique, 2018) }\end{array}$ & Peru & cuantitativa & $\begin{array}{c}\text { Cuestionario(Docentes y } \\
\text { estudiantes) }\end{array}$ \\
\hline
\end{tabular}


Referente a la figura 4, los instrumentos aplicados para el recojo de datos, los autores optaron por emplear la encuesta. De estos, cuatro (4) encuestas para docentes, cuatro (4) para estudiantes, uno (1) utilizó cuestionario y entrevista a docentes y estudiantes, finalmente tres (3) trabajaron con cuestionarios, entrevistas, observaciones y análisis a docentes.

Figura 4. Instrumento Usado

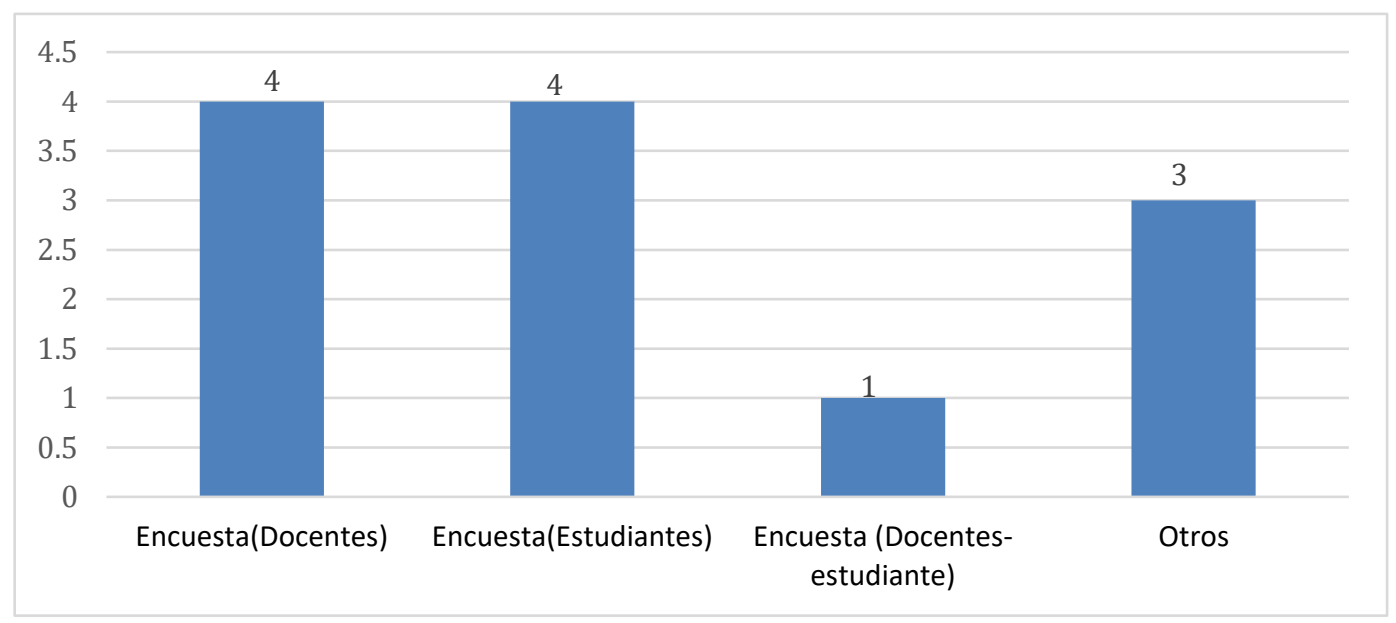

1.2 Análisis de las premisas conceptuales sobre Educación Ambiental

Dentro de los doce (12) investigaciones, se tiene a cuatro (4) estudios que conceptualizan a la EA como proceso educativo integral, porque motiva hacia el logro de conocimientos a fin de obtener un comportamiento equilibrado, del hombre hacia el medio ambiente, construyendo una mejor calidad de vida en la actual generación (Calderón et al., 2019; Espinoza y ortiz,2021; Henao \& Sánchez,2019; Oliveira et al., 2020).Así mismo, se puede observar cuatro(4) autores que refieren la EA como la base para el desarrollo sostenible, debido a que incentiva aptitudes, actitudes y valores sobre conservación ambiental de manera responsable, mitigando gran porcentaje de los problemas ambientales (Demoly y Dos santos,2018; Miyazaki et al., 2020; Alva,2019 ; Gonzales y Manrique, 2018).

En otro ámbito, tenemos dos (2) estudios de Barrero (2020) y Prosser et al., (2020) quienes mencionan a la EA, como pilar para el desarrollo de capacidades en temas ambientales. Así, Aparecida y Carlos (2018) consideran que la EA, consiste en las prácticas manipuladas a través de la formación, lo que se trasformará en la conservación de la naturaleza. Se demuestra en la tabla 3.

\section{Tabla N ${ }^{\circ}$. Definición de autores sobre Educación Ambiental}

\begin{tabular}{|c|l|}
\hline $\begin{array}{c}\text { AUTOR Y } \\
\text { AÑO }\end{array}$ & \multicolumn{1}{c|}{ DEFINICIÓN } \\
\hline $\begin{array}{c}\text { ( Calderon et } \\
\text { al., 2019) }\end{array}$ & $\begin{array}{l}\text { La EA es un proceso educativo que motiva hacia el desarrollo de } \\
\text { capacidad a fin de comparar aquellas actividades del hombre sobre } \\
\text { el medio ambiente. }\end{array}$ \\
\hline $\begin{array}{c}\text { (Demoly y Dos } \\
\text { santos,2018) }\end{array}$ & $\begin{array}{l}\text { EA también es definida como la cognición inventiva, las formas de } \\
\text { representar y conservar lo que queremos vivir. }\end{array}$ \\
\hline $\begin{array}{c}\text { (Espinoza y } \\
\text { Ortiz,2021) }\end{array}$ & $\begin{array}{l}\text { Refiere que la EA, como aquel proceso educativo integral que a la } \\
\text { larga genera no solo conocimientos, sino también actitudes, así como } \\
\text { valores y prácticas ambientalmente adecuadas. }\end{array}$ \\
\hline $\begin{array}{c}\text { (Miyazaki et } \\
\text { al., 2020) }\end{array}$ & $\begin{array}{l}\text { Define a la EA como la construcción del desarrollo sostenible, debido a } \\
\text { que promueve valores, actitudes y aptitudes necesarias en una } \\
\text { sociedad. }\end{array}$ \\
\hline
\end{tabular}




\begin{tabular}{|c|l|}
\hline $\begin{array}{c}\text { (Henao \& } \\
\text { Sánchez,2019) }\end{array}$ & $\begin{array}{l}\text { Hace énfasis a la EA como un proceso de formación que debería estar } \\
\text { presente con ejes objetivos, en todas las áreas con la finalidad de dar } \\
\text { coherencia y solidez }\end{array}$ \\
\hline $\begin{array}{c}\text { (Aparecida y } \\
\text { Carlos ,2018) }\end{array}$ & $\begin{array}{l}\text { consiste en una práctica social intencional, que brinda a los individuos } \\
\text { condiciones cognitivas para que, a través de acciones colectivas, } \\
\text { superen el modelo actual de sociedad, buscando cambios de nuestra } \\
\text { sociedad y en las relaciones con la naturaleza. }\end{array}$ \\
\hline $\begin{array}{c}\text { La EA es aquel Proceso que reconoce los valores con el objetivo de } \\
\text { implementar aquellas aptitudes y actitudes importantes, para } \\
\text { finalmente conocer la interrelación entre el individuo, cultura y } \\
\text { ambiente. }\end{array}$ \\
\hline $\begin{array}{c}\text { (Barrero,2020) } \\
\text { el EA considerada como pilar y base la cual permite } \\
\text { proceso educativo se puede realizar un cambio de pensamiento y } \\
\text { comportamiento para el cuidado del ambiente y la biodiversidad. }\end{array}$ \\
\hline $\begin{array}{c}\text { (Prosser et al., } \\
2020)\end{array}$ & $\begin{array}{l}\text { Describe a la EA como el desarrollo de capacidades pro ambientales } \\
\text { en una ciudadanía capaz de construir un mundo sustentable y justo } \\
\text { socialmente }\end{array}$ \\
\hline $\begin{array}{c}\text { (Oliveira et al., } \\
2020)\end{array}$ & $\begin{array}{l}\text { Es un proceso educativo cuyo propósito principal es insertar la } \\
\text { dimensión ambiental en la vida cotidiana de las personas. }\end{array}$ \\
\hline $\begin{array}{c}\text { (Rivas et al., } \\
2020)\end{array}$ & $\begin{array}{l}\text { Denominada como el escenario para la construcción de ideas de } \\
\text { transformación y cambio }\end{array}$ \\
\hline $\begin{array}{c}\text { (Gonzales y } \\
\text { Manrique, } \\
2018)\end{array}$ & $\begin{array}{l}\text { Definida como los conocimientos y aprendizajes, comportamientos y } \\
\text { prácticas para participar responsable y eficazmente en la mitigación } \\
\text { de los problemas ambientales. }\end{array}$ \\
\hline
\end{tabular}

Como se evidencia, en los estudios sobre EA, gran parte de los autores, la definen como un proceso educativo y base desarrollo sostenible en ese sentido, podemos deducir que las Instituciones Educativas, es el escenario para construir ideas, conocimientos y transformación en los estudiantes, en tanto se puede realizar nuevas perspectivas para el cuidado ambiental sustentable justo.

\subsection{Análisis de los resultados obtenidos}

A partir de la revisión de los doce (12) estudios, se arrojan varios resultados sobre Educación Ambiental en Instituciones Educativas. Destacando primordialmente la importancia que tiene la EA, no solo en lo académico sino también en la vida personal.

\section{Tabla $N^{\circ}$ 5. Análisis de resultados de artículos sobre Educación Ambiental}

\begin{tabular}{|l|l|}
\hline AUTOR Y AÑO & \multicolumn{1}{|c|}{ RESULTADO } \\
\hline (Barrero,2020) & $\begin{array}{l}\text { Encuesta a docentes de una II.EE , concluyeron que existen algunos } \\
\text { vacíos en los temas ambientales }\end{array}$ \\
\hline $\begin{array}{l}\text { (Demoly y Dos } \\
\text { Santos,2018) }\end{array}$ & $\begin{array}{l}\text { Talleres a Docentes y estudiantes donde concibieron de distinta } \\
\text { manera la EA. }\end{array}$ \\
\hline $\begin{array}{l}\text { (Miyazaki et al., } \\
\text { 2020) }\end{array}$ & $\begin{array}{l}\text { Encuesta y entrevista se demostró que la EA está delimitada por } \\
\text { poco conocimiento de los educadores. }\end{array}$ \\
\hline $\begin{array}{l}\text { (Calderon et } \\
\text { al., 2019) }\end{array}$ & $\begin{array}{l}\text { Concluye que las actividades vinculadas a la EA se encuentran } \\
\text { rezagadas en otros temas. }\end{array}$ \\
\hline
\end{tabular}




\begin{tabular}{|c|c|}
\hline $\begin{array}{l}\text { (Gonzales y } \\
\text { Manrique, 2018) }\end{array}$ & $\begin{array}{l}\text { El } 63,3 \% \text { de profesores conocen sobre temas ambientales sin } \\
\text { embargo el } 24 \% \text { de estudiantes desconoce }\end{array}$ \\
\hline $\begin{array}{l}\text { (Oliveira et al., } \\
2020)\end{array}$ & $\begin{array}{l}\text { El } 55 \% \text { de los maestros abordan la EA en tanto, un } 60 \% \text { de } \\
\text { estudiantes desconoce. }\end{array}$ \\
\hline $\begin{array}{l}\text { (Espinoza y } \\
\text { Ortiz,2021) }\end{array}$ & $\begin{array}{l}\text { A nivel de provincias en Abancay los estudiantes lo aborda la EA en } \\
\text { un } 55 \% \text {, mientras que Andahuaylas en un } 33 \% \text { y Chincheros con } \\
\text { un } 17 \% \text {. }\end{array}$ \\
\hline (Alva,2019) & $\begin{array}{l}\text { Encuesta a estudiantes de secundaria donde el } 58,7 \% \text { (54) } \\
\text { menciona como buena la EA. }\end{array}$ \\
\hline $\begin{array}{l}\text { (Aparecida y } \\
\text { Carlos ,2018) }\end{array}$ & $\begin{array}{l}\text { Sus resultados mostró que los docentes ejecutan la EA a través de } \\
\text { las políticas públicas y los proyectos ambientales. }\end{array}$ \\
\hline $\begin{array}{l}\text { (Prosser et al., } \\
2020)\end{array}$ & $\begin{array}{l}\text { Encuesta a } 150 \text { estudiantes con edades entre } 6 \text { y } 14 \text { años, pudo } \\
\text { detectar dificultades, vacíos y poca práctica en EA. }\end{array}$ \\
\hline $\begin{array}{c}\text { (Henao \& } \\
\text { Sánchez,2019) }\end{array}$ & $\begin{array}{l}\text { Los resultados muestran brecha entre en el diseño curricular nacional } \\
\text { y su concreción práctica en las instituciones educativas. }\end{array}$ \\
\hline $\begin{array}{l}\text { (Rivas et al., } \\
\text { 2020) }\end{array}$ & $\begin{array}{l}\text { Demostraron que los docentes construyen la EA con habilidades } \\
\text { institucionales. }\end{array}$ \\
\hline
\end{tabular}

Como se muestra en la tabla 5, según investigaciones concluyeron que existen algunos vacíos en los temas ambientales en las Instituciones Educativas (Barrero, 2020). Al respecto, Demoly y Dos S, (2018) como resultado, determinaron que docentes y estudiantes conciben de distinta manera la educación ambiental.

En esa misma línea Miyazaki et al., (2020) concluye que la EA, en las instituciones educativas estudiadas, está delimitada debido al poco conocimiento de los educadores, sin embargo, los estudiantes le dan la importancia debida con las distintas y pocas actividades aplicadas por los maestros.

Por otro lado,se evidenció que las actividades vinculadas a la EA se encuentran rezagadas en otros temas ( Calderón et al., 2019).Así mismo, Gonzales y Manrique,(2018) en su artículo mostraron que $63,3 \%$ de profesores conocen sobre temas ambientales, pero existe bajo nivel de conocimientos de alumnos con el $24 \%$, a pesar que existe la transversalidad en temas de EA en programas educativos. Para Oliveira et al., (2020), cerca del 55\% de los maestros abordan con frecuencia la EA en sus aulas, en tanto, pese al esfuerzo, se denota el desinterés por parte de los estudiantes con un $60 \%$.
A ello se suma la investigación de Espinoza y Ortiz, (2021) quienes demuestran que existe poca ejecución y logros en temas sobre educación ambiental, a nivel de tres (3) provincias las II.EE de Abancay aborda la EA en un 55\%, mientras que Andahuaylas lo ejecuta en un $33 \%$ y esta a su vez mayor que Chincheros que ocupa un 17\% . Alva, (2019) en sus resultados descriptivos, a estudiantes del nivel secundario, obtuvo que 58,7\% (54) menciona como buena la educación ambiental, en tanto demuestra que existe influencia entre coeficiencia y educación ambiental. Así, para Aparecida y Carlos (2018), sus resultados mostraron que las Instituciones Educativas desarrollan o ejecutan los procesos a través de las políticas públicas en educación ambiental en donde involucra lo transversal, interdisciplinariedad y los proyectos ambientales.

Finalmente, (Procer et al, 2020) a través de encuesta a 150 estudiantes con edades entre 6 y 14 años, pudo detectar dificultades, vacíos y poca práctica en EA en las aulas, ya que los mismos estudiantes mencionaron una serie de problemáticas ambientales en toda la Institución Educativa.

\section{CONCLUSIÓNES}

1. De acuerdo con la descripción de las investigaciones de Educación ambiental en Instituciones Educativas, se cuenta con un bajo índice de estu- 
dios y aportes debido a que presenta pocas publicaciones en la base de datos seleccionadas, como Scopus, Ebsco, cielo y Dialnet, comprendida entre los años 2018 al 2021, siendo en su mayoría de procedencia colombiana y gran parte de los enfoques desarrolladas con el método cualitativo con encuestas y entrevistas.

2. Por otra parte, referente a estudios de las premisas conceptuales se puede concluir que la educación ambiental es denominada como el proceso educativo sistemático, porque conlleva no solo al logro de conocimientos, sino también a su ejecución en la práctica a través de los proyectos educativos con soporte técnico de los maestros, dando paso a la concientización con un comportamiento debidamente equilibrado y valorado por los habitantes hacia su ambiente, por ello las aulas, es el espacio para instruir hacia la transformación en los estudiantes y perdure en el tiempo. 3. Por último, se identificó, que los educadores de las Instituciones Educativas cuentan con excelentes niveles de actitudes positivas hacia el ambiente, así lo demuestra el alto nivel de porcentaje en las investigaciones, sin embargo, su fortalecimiento se encuentra latente, entonces es factible perfeccionar la EA, así evitar barreras al momento de la formulación y transmisión de enseñanza a los estudiantes, siendo vital para la construcción de una sociedad altamente sostenible.

\section{REFERENCIA BIBLIOGRÁFICAS}

Al-Naqbi, A. \& Alshannag, Q.(2018).The status of Education for Sustainable development and sustainability knowledge, attitudes, and behaviors of UAE University students. International Journal of Sustainability in Higher Education, 19 (3), 566588. https://doi.org/10.1108/ijshe-06-2017-0091

Alva,W.(2019). Ecoeficiencia: Nueva estrategia para la educación ambiental en instituciones educativas. Investigación valdizana,24(2),77-84. https://doi.org/10.33554/riv.13.2.233

Aparecida, M., y Santana, L.(2018). Políticas públicas de educação ambiental e processos de mediação em escolas de Ensino Fundamental. Ciência \& Educação (Bauru),24(3),599-619. https://doi.org/10.1590/1516-731320180030005

Armesto, M. S., \& Montes de Oca, H.(2020). La gestión del talento humano en la sostenibilidad ambiental: Revisión Sistemática de artículos científicos disponibles acceso abierto en español. Anuario Facultad De Ciencias Económicas Y Empresariales, 57-67. https://anuarioeco.uo.edu. cu/index.php/aeco/article/view/5152

Arriola, C. (2017). La Educación y desarrollo de la conciencia Ambiental en estudiantes de Ingeniería Civil de la Universidad César Vallejo. Campus, 22(24), https://www.aulavirtualusmp. pe/ojs/index.php/rc/article/view/1267

Barrero, J. E. (2020). The Importance of Environmental Education in Basic and High School Students in Three Public Educational Institutions in El Espinal (Tolima); La Importancia de la Educación Ambiental en Estudiantes de Básica y Media en Tres Instituciones Educativas Públicas en El Espinal (Tolima). Miradas, 1(3), 129 - 142 https://doi. org/10.22517/25393812.24473

Berdugo, N. y Montaño, W. (2017). La Educación Ambiental en las Instituciones de educación superior públicas en Colombia. Revista Científica General José María Córdova, 15(20), 127-136. http://dx.doi.org/10.21830/19006586.178

Calderon, J. E., Suarez, E. A., Atencio, F. A., Blanco, E. P., Oyaga, R., \& Tejera, M. P. (2019). Applicability of the national policy of environmental education in educational institutions. [Análisis de la aplicabilidad de la Política Nacional de Educación Ambiental en las instituciones educativas] Espacios, 40(29), 1-8 http://www.revistaespacios.com/a19v40n29/ a19v40n29p01.pdf

Cóndor, E. (2018). Dimensión ambiental en la formación profesional de los estudiantes de la Facultad de Educación dela Universidad Nacional 
de Huancavelica. Educación, 27(53), 41-56. https://doi.org/10.18800/educacion.201802.003

Demoly, K. R., \& Dos Santos, J. S. (2018). Learning, environmental education and school: Ways of en-acting in the experience of students and teacher. Ambiente e Sociedade, 21 doi:10.1590/1809-4422asoc0087r2vu1811ao

Espejel, A y Flores.A. (2017). Successful experiences of environmental education in the young high school students in tlaxcala, México. Revista Luna Azul, 1(44). https://www.redalyc. org/articulo.oa?id=321750362018

Espinoza, F. A., \& Ortiz, J. I. (2021). Percepción docente sobre la aplicación del enfoque ambiental en instituciones educativas de Apurímac, Perú. Conrado, 17(79),133-140. http://scielo.sld. $\mathrm{cu} /$ scielo.php? script $=$ sci_arttext\&pid $=$ S1990 86442021000200133\&lng=es\&tlng=es.

Estrada, E., Huaypar, K. y Mamani, H. (2020). La Educación Ambiental y el manejo de residuos sólidos en una institución educativa de Madre de Dios, Perú. Ciencia Amazónica (Iquitos), 8(2), 239 - 252. https://doi.org/10.22386/ca.v8i2.300

Estrada, E., Mamani, H., \& Huaypar , K. (2020). Eficacia del programa Cuidemos el Ambiente en el desarrollo de la conciencia ambiental de estudiantes de educación primaria en Madre de Dios, Perú. Ciencia Amazónica (Iquitos), 8(1), https://doi.org/10.22386/ca.v8i1.282

González Manrique de Lara, T., \& Manrique de Lara Suarez, L. (2019). Educación ambiental en las instituciones educativas de secundaria del distrito José Crespo y Castillo, provincia de Leoncio Prado. RevIA,7(4), 22-7 http://revistas. unas.edu.pe/index.php/revia/article/view/139

Henao, O., \& Sánchez, L. (2019). La educación ambiental en Colombia, utopía o realidad. Conrado, 15(67),213-219.

http://scielo.sld.cu/scielo.php?script $=$ sci_ arttext\&pid=S1990-86442019000200213\&lng=e s\&tlng=es.
Hernan M., Escobar, G., Luna.,c, \& Moren., A. (2021). La transversalidad de la educación ambiental en dos instituciones educativas del departamento de Nariño, Colombia. Revista Boletín Redipe, 10(5). https://doi.org/10.36260/ rbr.v10i5.1300

Herrera, D. y Ríos, D. (2017). Educación ambiental y cultura evaluativa. Algunas reflexiones para la construcción de eco-consciencias. Estudios Pedagógicos, 43(1), 389-403. http://dx.doi. org/10.4067/S0718-07052017000100022

Mamani, H., Estrada, E., Gallegos, N. y Huaypar, K. (2020). Actitudes hacia la conservación ambiental en adolescentes de educación secundaria en Madre de Dios, Perú. Ciencia Amazónica (Iquitos), 8(1), 99 - 110. https://doi. org/10.22386/ca.v8i1.283

Márquez, D., Hernández, A., Márquez, L. y Casas, M. (2021). La educación ambiental: evolución conceptual y metodológica hacia los objetivos del desarrollo sostenible. Revista Universidad y Sociedad, 13(2), 301-310. https://rus.ucf.edu.cu/ index.php/rus/article/view/1968

Ministerio de Educación (2016) Plan Nacional de Educación Ambiental 2017-2022(PLANEA). http://extwprlegs1.fao.org/docs/pdf/ per161555anx.pdf

Miyazaki, M., Oxilia -Dávalos, V.E., \& LeivaEnrique, M. G. (2020). La educación ambiental relacionada al desarrollo sostenible en las instituciones educativas del municipio de Villa Hayes. Reportes científicos de la Facen, 11(2), 14-19. https://dx.doi.org/10.18004/ rcfacen2020.11.02.14

Oliveira, L.C., Queiroz, G.F., y Costa, P. (2020). El enfoque de la educación ambiental en la relación maestro-alumno en las escuelas públicas de Parauapebas en el Estado de Pará, Brasil. Investigación, Sociedad y Desarrollo; 9 (12), 2525-3409.https://doi.org/10.33448/rsdv9i12.10551 
Peramo,P.(2020) Cómo elaborar una revisión sistemática. Revista de Investigación Educativa,20(01).

https://dx.doi.10.13140/RG.2.2.31465.85608

Pérez, N., Cleveland, M., Lleras, S. y Cortés, N. (2019) educación ambiental mediante la metodología aprendizaje-servicio: percepción de adquisición de competencias e impacto en la comunidad. Revista Universidad y Sociedad, 11(4), 154-162. http://scielo.sld.cu/ scielo.php?script $=$ sci_arttext \&pid $=$ S22 18 36202019000400154\&lng=es\&tlng=es.

Prosser, G., Salazar, M. S., Perez, S., Perez, M., \& Prosser, C. (2020). Evaluacion de un programa de educacion ambiental desde la voz del alumnado/ Evaluation of an environmental education program through the voices of students/Avaliacao de um programa de educacao ambiental desde a voz dos alunos. Revista Latinoamericana de Ciencias Sociales, Ninez y Juventud, 18(2), https://doi.org/10.11600/1692715x.18206

Pulido, V. y Olivera, E. (2018) Pedagogical contributions to environmental education: a theoretical perspective. Revista de Investigaciones Altoandinas, 20(3). https://dx.doi.org/10.18271/ ria.2018.397

Quintana, A. (2017) La educación ambiental y su importancia en la relación sustentable: HombreNaturaleza-Territorio. Revista Latinoamericana de ciencias sociales, niñez y juventud, 15(2). https://link.gale.com/apps/doc/A524752944/ $\mathrm{AONE} ? \mathrm{u}=\mathrm{univcv} \& \mathrm{sid}=\mathrm{AONE} \& \mathrm{xid}=819994 \mathrm{~cd}$.

Rivera, M. (2017). La Educación Ambiental en Puerto Rico: propuesta para un modelo interdisciplinario de educación formal, Runae, (1), 167-184. https://revistas.unae.edu.ec/index. php/runae/article/view/167

Salvatierra, E. (2018) Environmental dimension in the professional training of the students of the Education Faculty of the National University of Huancavelica. Educación,27(53).
https://link.gale.com/apps/doc/A562690619/

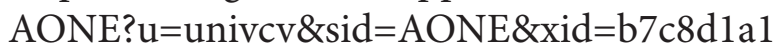

Silva, M., Lopes, M. y Guillen, S. (2019) Educación ambiental para el desarrollo sostenible: enfoque desde San Luís, Santiago de Cuba. Revista Brasileira de Estudos Pedagógicos, 100 (255), 501-516.

https://doi.org/10.24109/2176-6681. rbep.100i255.4051

Yangali, V., Vasquez, T., Huaita, A y Baldeon,M. (2021). Comportamiento ecológico y cultura ambiental, promovido a través de la educación virtual en estudiantes de Lima-Perú. Revista de Ciencias Sociales, 27(1),1-15.

h t t p s : / / w w w. r e d a l y c.org / journal/280/28065533031/movil/ 